\title{
Atypical Intrapartum Maternal Heart Rate Pattern in a Woman with Paroxysmal Supraventricular Tachycardia
}

\author{
Junsuke Muraoka ${ }^{1}$, Masanao Ohhashi², Hiroshi Sameshima1 \\ ${ }^{1}$ Department of Obstetrics and Gynecology and Center for Perinatal Medicine, University of Miyazaki, Kihara-Kiyotake, Miyazaki, \\ Japan \\ ${ }^{2}$ Department of Obstetrics and Gynecology, Miyazaki Medical Association Hospital, Arita, Miyazaki, Japan \\ Email: `jyunsuke_muraoka@med.miyazaki-u.ac.jp
}

How to cite this paper: Muraoka, J., Ohhashi, M. and Sameshima, H. (2021) Atypical Intrapartum Maternal Heart Rate Pattern in a Woman with Paroxysmal Supraventricular Tachycardia. Open Journal of Obstetrics and Gynecology, 11, 1589-1595. https://doi.org/10.4236/ojog.2021.1111148

Received: October 21, 2021

Accepted: November 21, 2021

Published: November 24, 2021

Copyright (c) 2021 by author(s) and Scientific Research Publishing Inc. This work is licensed under the Creative Commons Attribution International License (CC BY 4.0).

http://creativecommons.org/licenses/by/4.0/

\begin{abstract}
Pregnant women show characteristic hemodynamics, and their heart rates ordinarily increase with uterine contractions during labor. Conversely, uterine contraction-associated decelerations of maternal heart rate (MHR) are rare. We present a pregnant woman with paroxysmal supraventricular tachycardia (PSVT) who exhibited intrapartum MHR deceleration pattern. We performed simultaneous fetal heart rate monitoring with an external ultrasound transducer and MHR monitoring with a tocogram during her parturition. She developed a PSVT exacerbation in the second stage of parturition. As revealed by cardiotocography, the MHR baseline abruptly declined at onset of uterine contractions during the active phase of labor. Recovery followed, and the contraction resolved. The tachycardia got prolonged as the labor advanced. Our patient exhibited the unusual, up-and-down changes of the MHR pattern associated with labor contractions. The etiology of the occurrence of uterine contraction-associated MHR decelerations was unclear in the present case. Continuous tracing to visually inspect MHR patterns during parturition is a simple method for examining real-time MHR status of patients treated within obstetric practice settings.
\end{abstract}

\section{Keywords}

Heart Rate, Labor, Paroxysmal Supraventricular Tachycardia, Pregnancy, Uterine Contraction

\section{Introduction}

Maternal heart rate (MHR) monitoring during labor has some benefits. In 
women with arrhythmias and congenital heart diseases, MHR monitoring is necessary [1]. Intrapartum MHR pattern can be a pragmatic marker of intrapartum volume status and can provide information about intrapartum fluid management [2]. MHR monitoring also helps avoid misinterpretation of MHR as a fetal heart rate (FHR) during labor, as we previously reported [3].

During parturition, maternal hemodynamics change considerably before and after uterine contractions by compensatory mechanisms. The MHR usually increases on uterine contractions in low-risk pregnancies [4], whereas some investigators have reported a small reduction in intrapartum MHR with uterine contraction [5] [6]. However, the association of MHR change with labor in pregnant women with paroxysmal supraventricular tachycardia (PSVT) is poorly understood. This report examined how the MHR pattern of a pregnant woman with PSVT changed in response to labor using continuous heart rate tracing during parturition. The patient provided written informed consent for this report.

\section{Case Presentation}

A 27-year-old primiparous Japanese woman visited our hospital to evaluate her arrhythmia at 22 gestational weeks (GW). She had no family history of heart disease or sudden death. At the age of 20, she was diagnosed with PSVT after a first episode of palpitation and was administrated verapamil. Since then, because her symptoms improved, she stopped the medication. When she visited our hospital, cardiac ultrasonography revealed no structural abnormalities or circulatory shunts with normal cardiac contractility. A Holter electrocardiographic monitoring performed at 22 and $39 \mathrm{GW}$ showed sporadic atrioventricular nodal reentrant tachycardia with a maximum duration of $16 \mathrm{~min}$ and a maximum of 2 min, respectively. Her pregnancy proceeded unremarkably and without advanced symptoms of cardiac features until term, as shown in Table 1. In discussion with a cardiologist, we equipped direct-current defibrillation apparatus and adenosine triphosphate disodium readily available in preparation for hemodynamic instability during labor, because severe maternal morbidity was most associated with the pregnancy with PSVT. At 39 and 2/7 GW, she was hospitalized with labor onset. Her admission hemoglobin level was $11.9 \mathrm{~g} / \mathrm{dL}$ and 77 beats/min of HR. We performed simultaneous FHR monitoring with an external ultrasound transducer and MHR monitoring with a tocogram (Model FM-30, Atom Medical, Inc., Tokyo, Japan). She received fluid replacement intravenously and permitted to take oral rehydration. After careful evaluation of the potential benefits and risks for epidural anesthesia, she wished for natural childbirth without epidural analgesia. The MHR until the cervix dilated $<6 \mathrm{~cm}$ remained constant at a normal baseline level and rhythm (Figure 1(A)), and she had no complaints of labor pains. The cervical dilation progressed as contractions increased. After the cervix dilated $\geq 6 \mathrm{~cm}$, the MHR level was $>180$ beats $/$ min during the dormant period of every uterine contraction and abruptly returned to normal once uterine contractions started (Figure 1(B)). She developed painful labor but could 

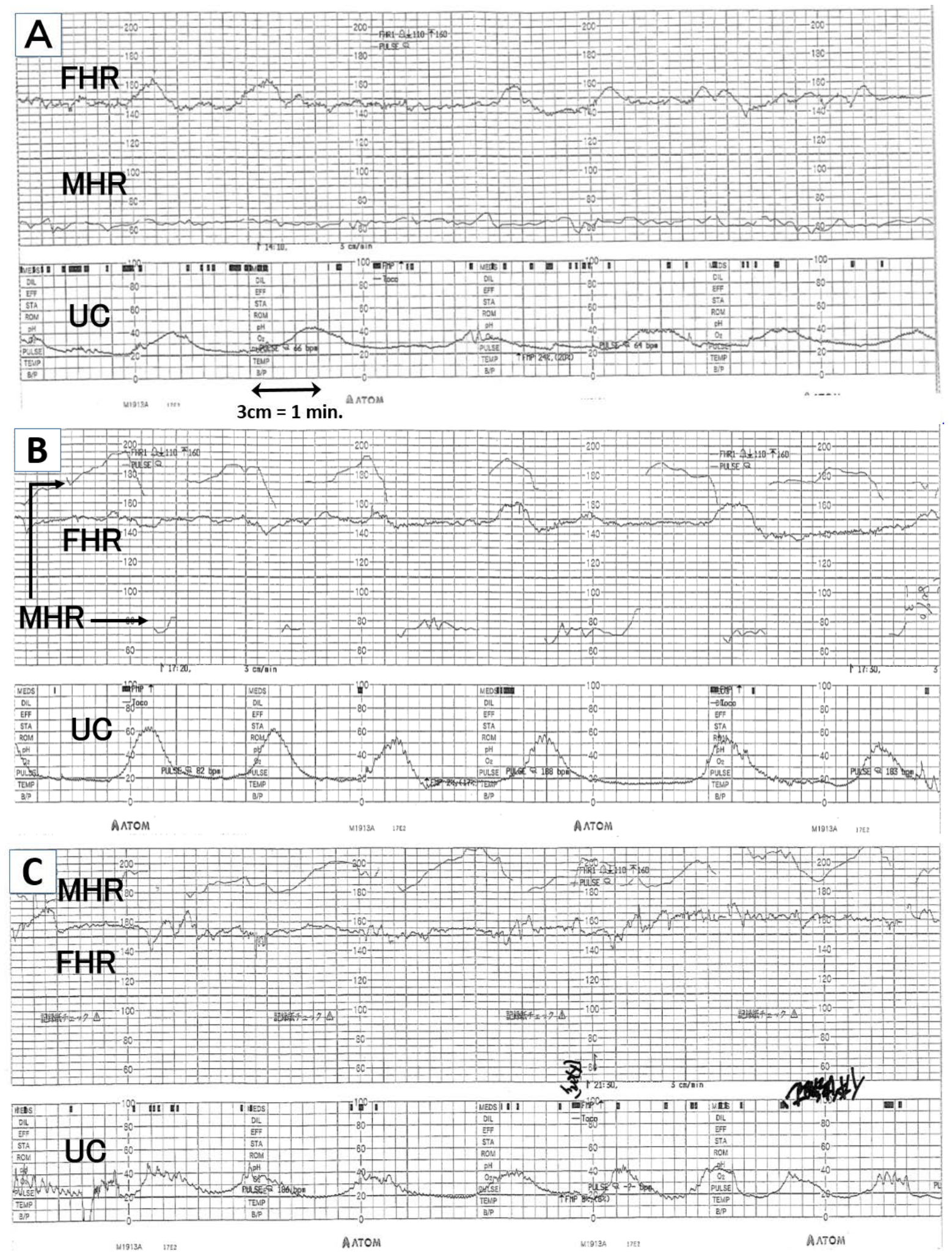

Figure 1. Intrapartum FHR and MHR tracing charts. MHR constantly showed a normal rhythm during the former phase of labor (A). In the latter phase of labor, MHR declined. This was accompanied by uterine contraction onset and soon recovered (B). Finally, MHR surpassed 200 beats/min, and the MHR baseline level intermittently declined to 180 beats/min during uterine contractions just before delivery (C). MHR, maternal heart rate; FHR, fetal heart rate; UC, uterine contraction. 
Table 1. Temporal course of cardiac symptom, measurements, and therapeutic approach of the patient.

\begin{tabular}{|c|c|c|c|c|}
\hline & Symptom & Holter ECG records & Ultrasonography findings & Treatment \\
\hline \multicolumn{5}{|l|}{ Preconception } \\
\hline 20-year-old & Pulpitation, infrequently & PSVT & Normal & Oral verapamil for a month \\
\hline \multicolumn{5}{|l|}{ During pregnancy } \\
\hline $22 \mathrm{GW}$ & Pulpitation, sometimes & $\begin{array}{c}\text { Sporadic AVNRT } \\
\text { with a maximum duration } \\
\text { of } 16 \text { minutes }\end{array}$ & FS $30 \%$, EF $54 \%$ & Free of medication \\
\hline $30 \mathrm{GW}$ & Pulpitation, every day & Not recorded & FS 39\%, EF 66\% & Free of medication \\
\hline $39 \mathrm{GW}$ & Pulpitation, every day & Only 2 minutes' AVNRT & FS $32 \%$, EF $52 \%$ & Free of medication \\
\hline \multicolumn{5}{|l|}{ Intrapartum } \\
\hline 1st stage of labor & $\begin{array}{l}\text { None } \\
\text { Pulpitation }\end{array}$ & Not recorded & Not measured & $\begin{array}{l}\text { Vagal maneuvers, and } \\
\text { equipment of intravenous } \\
\text { adenosine and direct- } \\
\text { current cardioversion }\end{array}$ \\
\hline \multicolumn{5}{|l|}{ Postpartum } \\
\hline $\begin{array}{c}\text { One month after } \\
\text { childbirth }\end{array}$ & Pulpitation, infrequently & Normal & FS $36 \%$, EF $60 \%$ & Free of medication \\
\hline
\end{tabular}

Abbreviations: GW, gestational weeks; ECG, electrocardiogram; PSVT, paroxysmal supraventricular tachycardia; AVNRT, atrioventricular nodal reentrant tachycardia; FS, fractional shortening; EF, ejection fraction.

maintain deep breathing without performing the Valsalva maneuver. After the second stage of labor (fully dilated cervix), the MHR baseline level finally surpassed 200 beats/min, and the MHR baseline level intermittently declined to 180 beats/min during uterine contractions (Figure $1(\mathrm{C})$ ). She performed several times of Valsalva pushing and spontaneously delivered without forceps or vacuum extraction. After a healthy female, 3198-g neonate was born, the maternal tachycardia lasted for about $20 \mathrm{~min}$ and then recovered to a normal HR of 60 beats/min. No temperatures higher than $38.0^{\circ} \mathrm{C}$ or urinary ketones were measured during delivery. The periodic tachycardia appeared for several days after childbirth and then disappeared.

\section{Discussion}

Here we report a case of a pregnant woman with PSVT who exhibited intrapartum MHR deceleration patterns. Our findings demonstrated that the MHR transiently declined upon onset of uterine contractions, and the tachycardia duration increased as labor progressed after the cervix dilated $\geq 6 \mathrm{~cm}$. There have no previous reports on how MHR changes during contraction in women with PSVT. The up-and-down changes of the MHR pattern seem curious phenomena.

The human body features various heart rate regulatory mechanisms. In pregnant women, uterine contractions increase the circulatory volume, and both cardiac output (CO) and blood pressure usually increase during labor [7]. CO during the first and the second stage of labor increases up to $30 \%$ and by as 
much as $50 \%$, respectively [8]. The Bainbridge reflex, an atrial sympathetic-mediated response, increases MHR. However, the Valsalva maneuver, in which $\mathrm{CO}$ is reduced due to increasing chest pressure that results from maternal bearing down, can decrease MHR. The Bainbridge reflex acts in opposition to the Valsalva maneuver.

It is well known that MHR increases on uterine contraction [4]. Our previous research revealed that MHR significantly increased during labor [3]. An observational study by Kuhn et al. demonstrated that MHR increased by $52 \%$ during the second stage of labor [9]. These changes may be caused by the increased stroke volume and catecholamine levels during uterine contraction [6]. Conversely, our case exhibited atypical MHR changes in response to uterine contractions. As seen in Figure 1(B), the MHR baseline abruptly declined when the uterine contractions started and recovered with the contraction resolved. The tachycardia duration was prolonged as the patient entered the latter phase of labor. Finally, the tachycardia persisted until delivery, as described in Figure 1(C).

MHR deceleration associated with uterine contraction is rare and remains unexplained [10]. The mechanistic variations of MHR are the product of baroreceptor reflexes, including the Bainbridge reflex and Valsalva maneuver; however, we do not know what reflex is preferentially activated when a uterine contraction begins. First, Valsalva pushing and spontaneous pushing have different effects on the MHR. When the Valsalva pushing technique is used, the woman is instructed to take a deep breath when the contraction begins, then hold her breath, and push as long and hard as she can in synchrony with her contractions. On contrast, the spontaneous pushing approach is a self-directed bearing down technique in response to an involuntary urge. Here, the push starts from a resting respiratory volume, without first taking a deep breath. Because attendant physicians or midwives at our hospital encourage almost parturient patients to perform Valsalva pushing only before delivery, there is no clear explanation for the MHR deceleration exhibited by this case. Second, we speculated that maternal dehydrated status might serve as decreasing the MHR. Lappen et al. analyzed the characteristics between contraction-associated MHR deceleration and intrapartum maternal volume status in healthy women [2]. They found that a contraction-associated MHR deceleration pattern is three-fold more frequent among hypovolemic women when compared with its occurrence in euvolemic women. If our patient had become dehydrated during labor despite being negative urinary ketones, the blood volume may have been inadequate to generate the Bainbridge reflex. In that way, MHR transiently decreased because of Valsalva pushing without MHR acceleration after the Bainbridge reflex. Further case analyses will probably be needed to confirm the mechanism of MHR regulation in pregnant women with PSVT.

Arrhythmias are the most common cardiac complication encountered during pregnancy. In previous reports, 50\% - 85\% of women with onset of PSVT before their pregnancies had arrhythmia exacerbations during their pregnancies [11] [12]. In the present case, we could observe the development of a PSVT exacerba- 
tion during parturition and recognized the duration of the arrhythmia by continuously monitoring MHR and FHR. Antiarrhythmic drugs are often reserved to treat arrhythmias associated with neither clinically significant symptoms nor compromised hemodynamics. Following the cardiovascular expert advice, we were therefore able to observe the natural course of MHR patterns without using any antiarrhythmic drugs during parturition. In general, epidural anesthetic care for labor and vaginal delivery should be provided to women with ventricular tachyarrhythmia. Even though the patient progressed without symptoms of arrhythmia, the benefits of epidural analgesia should have been fully explained. Continuous MHR recording was useful for understanding the arrhythmia duration and its baseline heart rate, which performed alike in an electrocardiogram monitored setting. Interpretation of the real-time MHR tracing seems to be a simplified method in obstetric practices.

\section{Conclusion}

The atypical MHR pattern, consisting of transiently declined synchronously with uterine contraction onset, and the tachycardia duration was prolonged as labor progressed. Continuous MHR recording during parturition is a simple method for examining real-time MHR status for PSVT patients treated within obstetric practice settings.

\section{Acknowledgements}

The authors thank Dr. H. Matsuura, Cardiologist of Miyazaki Medical Association Hospital for his clinical assistance and scientific advice.

\section{Authors' Contribution}

Junsuke Muraoka: data acquisition and drafting manuscript; Masanao Ohhashi: data acquisition and scientific comments; Hiroshi Sameshima: scientific comments and supervision.

\section{Conflicts of Interest}

The authors declare no conflicts of interest regarding the publication of this article.

\section{References}

[1] Romen, A., Romen, Y., Katz, M. and Battler, A. (2004) Incidence and Characteristics of Maternal Cardiac Arrhythmias during Labor. American Journal of Cardiolo$g y$, 93, 931-933. https://doi.org/10.1016/j.amjcard.2003.12.042

[2] Lappen, J.R., Chien, E.K. and Mercer, B.M. (2018) Contraction-Associated Maternal Heart Rate Decelerations: A Pragmatic Marker of Intrapartum Volume Status. $\mathrm{Ob}$ stetrics and Gynecology, 132, 1011-1017. https://doi.org/10.1097/AOG.0000000000002808

[3] Muraoka, J., Furukawa, S. and Sameshima, H. (2015) Maternal Heart Rate and Fetal Heart Rate Characteristics in Response to Labor. Jacobs Journal of Gynecology \& 
Obstetrics, 2, 12.

[4] Silverside, C.K. and Colman, J.M. (2007) Physiological Changes in Pregnancy. Second Edition, Blackwell Publishing, Massachusetts, 6-17.

https://doi.org/10.1002/9780470994955.ch2

[5] Lee, W., Rockey, R., Miller, J. and Cotton, D.B. (1989) Maternal Hemodynamic Effects of Uterine Contractions by M-Mode and Pulse-Doppler Echocardiography. American Journal of Obstetrics and Gynecology, 161, 974-977. https://doi.org/10.1016/0002-9378(89)90765-5

[6] Van Veen, T.R., Belfort, M.A. and Kofford, S. (2012) Maternal Heart Rate Patterns in the First and Second Stages of Labor. Acta Obstetricia et Gynecologica Scandinavica, 91, 598-604. https://doi.org/10.1111/j.1600-0412.2012.01371.x

[7] Duvekot, J.J. and Peeters, L.L. (1994) Maternal Cardiovascular Hemodynamic Adaptation to Pregnancy. Obstetrics and Gynecology Survey, 49, S1-S14. https://doi.org/10.1097/00006254-199412011-00001

[8] Ouzounian, J.G. and Elkayam, U. (2012) Physiologic Changes during Normal Pregnancy and Delivery. Clinical Cardiology, 30, 317-329.

https://doi.org/10.1016/j.ccl.2012.05.004

[9] Kuhn, J.C., Falk, R.S. and Langester, E. (2017) Haemodynamic Changes during Labour: Continuous Minimally Invasive Monitoring in 20 Healthy Parturients. International Journal of Obstetrical Anesthesia, 31, 74-83. https://doi.org/10.1016/j.ijoa.2017.03.003

[10] Sherman, D.J., Frenkel, E., Kurzweil, Y., Padua, A., Arieli, S. and Bahar, M. (2002) Characteristics of Maternal Heart Rate Patterns during Labor and Delivery. Obstetrics and Gynecology, 99, 542-547. https://doi.org/10.1097/00006250-200204000-00005

[11] Lee, S.H., Chen, S.A., Wu, T.J., Chiang, C.E., Cheng, C.C., Tai, C.T., et al. (1995) Effects of Pregnancy on First Onset and Symptoms of Paroxysmal Supraventricular Tachycardia. American Journal of Cardiology, 76, 675-678. https://doi.org/10.1016/S0002-9149(99)80195-7

[12] Silverside, C.K., Harris, L., Haberer, K., Sermer, M., Colman, J.M. and Siu, S.C. (2006) Recurrence Rates of Arrhythmias during Pregnancy in Women with Previous Tachyarrhythmia and Impact on Fetal and Neonatal Outcomes. American Journal of Cardiology, 97, 1206-1212. https://doi.org/10.1016/j.amjcard.2005.11.041 